challenging and potentially poorly completed. By standardizing available equipment and furthermore through practical steps such as separating the equipment required on a daily basis and that used less frequently the retention of equipment improved. This enables delivery of high quality, timely and thorough monitoring and assessment of physical health to be achievable.

Improving cardiometabolic screening on an inpatient psychiatric ward: a quality improvement project

Harrison Howarth*, Jonathan Pass, Fahel Ahmed and Sarah Wiethoff

Camden and Islington Foundation NHS Trust

${ }^{*}$ Corresponding author.

doi: 10.1192/bjo.2021.522

Aims. Primary aim: To increase the proportion of patients receiving a full cardiometabolic screen whilst on the ward to $75 \%$.

Secondary aims: To improve communication with GPs regarding cardiometabolic health, to improve the rates of intervention when abnormalities are found to $75 \%$.

Background. People with serious mental illness are known to have significantly increased risk of cardiometabolic syndrome than the general population. Estimates suggest there would be up to 12,000 fewer deaths from cardiovascular disease if people with serious mental illness had the same outcomes as the general population. People with serious mental illness die on average 20 years earlier than the general population due to preventable physical health problems.

Whilst on the ward, we have an excellent opportunity to screen and treat patients with cardiometabolic risk factors, yet screens are often incomplete, not acted upon, or simply not carried out. Method. Using the Plan-Do-Study-Act (PDSA) methodology, we trialed interventions to improve the cardiometabolic screening process on out 16 bed inpatient ward. Across 8 cycles, we set up a protocol to ensure all new patients received a full cardiometabolic screen during their admission reviews, engaged nursing staff with the process and managed inconsistencies with blood transportation and delivery. We also started using British Heart Foundation information leaflets, and treating patients in accordance with the Lester Tool: Positive Cardiometabolic Health Resource. We made design changes to the discharge summary template allowing for clear communication with GPs on discharge.

Result. At the end of 8 cycles, we had achieved $100 \%$ compliance with the full cardiometabolic screen (as defined by the Lester Tool) from a baseline of just $25 \%$. We also improved intervention with identified abnormalities from a baseline of $0 \%$ to $100 \%$.

Conclusion. Improvements in cardiometabolic screening and treatment were possible using the PDSA methodology. Given the success of this quality improvement project, we plan to introduce our methodology onto other wards in the trust.

Improving attitudes towards the COVID-19 vaccine in forensic mental health workers: a quality improvement project

Harrison Howarth*, Toby Stevens, Benjamin Gostick, Lisanne Stock and David Stone

North London Forensic Service

${ }^{\star}$ Corresponding author.

doi: 10.1192/bjo.2021.523
Aims. The primary aim of the project was to improve attitudes towards the COVID-19 vaccine in forensic mental health staff at a large regional tertiary forensic psychiatry unit. The main variable examined was attitudes towards safety of the vaccine. Secondary aims included decreasing misinformation about the vaccine and improving vaccine uptake.

Method. Paper questionnaires were distributed to willing staff members across 6 forensic inpatient wards within the North London Forensic Service. Participants included a range of allied health professionals including nurses, health care assistants, ward managers, occupational therapists, assistant therapists and administrative staff. Questionnaires used a mixture of Likert scale for agreement/disagreement with statements and yes/no questions.

Plan-Do-Study-Act (PDSA) methodology was utilised in implementing changes, and repeat questionnaires used to measure changes in attitude and behaviour. Change ideas implemented included the creation of 'mythbusters' posters which target vaccine misinformation, the creation and distribution of posters of staff members who had already taken their vaccine, the creation of vaccine champions to aid engagement in conversation about the vaccine, vaccine information packs being distributed to all wards and the opportunity for staff to 'drop-in' to clinics for information about the vaccine.

Result. Vaccine uptake improved from $7 \%$ before interventions to $69 \%$ after interventions.

The proportion of people very unlikely or unlikely to get the vaccine reduced from $25 \%$ to just $9 \%$. The proportion of those feeling neutral reduced from $32 \%$ to $6 \%$. The proportion of those either likely or very likely to get the vaccine increased from $34 \%$ to $85 \%$.

Before interventions only $20 \%$ felt that the vaccine was either safe or very safe. This improved to $63 \%$ after interventions

Before interventions, only $27 \%$ of respondents felt they had received enough information by the trust to make an informed decision. After interventions, $80 \%$ said they had received enough information.

The project was successful in reducing misinformation in every domain. Particularly reassuring was the reduction to zero of some of the most harmful misinformation claims, such as the presence of a tracking chip in the vaccine and the belief that COVID does not exist.

$71 \%$ of respondents indicated the interventions we set out changed their view on the COVID-19 vaccine.

Conclusion. The changes implemented lead to clear improvements in all domains measured, suggesting targeted information is an effective strategy in improving uptake and attitudes around the vaccination program.

A quality improvement project to improve attendance to the physical health clinic at Southwark team for early psychosis

Christina Huggins*, Joanna Cranshaw, Lucy Pauli and Beate Haege

South London and Maudsley NHS Foundation Trust ${ }^{\star}$ Corresponding author.

doi: 10.1192/bjo.2021.524

Aims. The aim of this project was to improve the booking and attendance of patients under Southwark Team for Early Psychosis (STEP) into the physical health clinic.

Background. STEP is an Early Intervention Service which provides treatment to 230 adults (18-65 years) with first episode 
psychosis in the community. In line with national and trust guidance, physical health checks are completed at baseline, 3 months, 6 months and annually, through a weekly physical health clinic run by the core trainee (CT). This is an essential opportunity to assess and monitor patients' physical health and aid decisions regarding psychotropic medications, which is particularly important given the increased morbidity and mortality in this group and their reduced engagement with health services. It was noted that attendance to the clinic was poor and there was no guidance about how to communicate the results to the General Practitioner (GP).

Method. Data on the number of clinic appointments booked and attended were collected over 3 defined 9 week intervals between $17 / 09 / 18$ and $29 / 07 / 19$. The interventions were implemented prior to the third round of data collection and included an educational session to the STEP team and a protocol for booking and running the clinic to be used by the CT. We devised a physical health questionnaire to be completed by patients on arrival, which includes a summary of the Maudsley guidelines for antipsychotic monitoring. Finally, we created a template letter to communicate the results to the GP.

Result. Following the interventions, the percentage of available clinic slots booked increased from an average of $27.8 \%$ to $100 \%$. The proportion of slots attended reduced from an average of $80 \%$ to $50 \%$. However, the absolute number of patients booked into clinic increased from an average of 10 patients over 9 weeks pre-intervention, of which an average of 8 patients attended, to 36 patients post-intervention, of which 18 attended. Conclusion. We observed full utilisation of available clinic slots post-intervention and an increase in the absolute number of patients attending. Given the ongoing use of the protocols developed, we expect these changes to be sustainable. The number of patients attending could be further increased by training additional staff to run the clinic more often and more flexibly. The number of Did-Not-Attends could be reduced by carecoordinators sending reminder texts prior to the appointments.

\section{Investigating sleep quality on an inpatient psychiatry ward}

Joshua Hughes $^{1 \star}$, Rhianne Thomas ${ }^{1}$, Jonathan Macklin ${ }^{1}$ and Jonathan Owen ${ }^{2}$

${ }^{1}$ NHS Dumfries and Galloway and ${ }^{2}$ University of Edinburgh ${ }^{*}$ Corresponding author.

doi: 10.1192/bjo.2021.525

Aims. Sleep is essential for optimal physiological functioning, but often interrupted in hospital settings. Disturbed sleep is associated with relapse of mood disorders and multiple comorbidities including impaired immunological function and increased cardiovascular risk. There are unique environmental challenges on psychiatry wards, such as overnight monitoring. Recent studies highlight the importance of evaluating and managing inpatient sleep disturbance. Aims include exploring the extent to which patients' sleep is impacted by inpatient admission, elucidating causes of sleep disturbance and determining ways to improve sleep during admission.

Method. Patients aged 18-65 years, who consented and were expected to be inpatients for a week, were approached after 72 hours of admission $(n=35)$. Quantitative and qualitative data, including on pre-hospital and hospital sleep quantity and quality, were gathered, as part of a cohort characterisation. Questionnaires using Pittsburgh Sleep Quality Index elements were used to gather data. Offering earplugs as a sleep-aid intervention was implemented, with sleep quantity and quality reassessed 72 hours post-intervention. In response to feedback, sound monitoring at regular intervals overnight was undertaken using a decibel-metre to determine noise baseline and variation.

Result. All patients approached agreed to participate. Pre-hospital average sleep quantity was 5.2 hours, with restedness score of 4.3 , and $71 \%$ patients rating their sleep as 'bad'. After 72 hours postadmission, average sleep length was 6.5 hours and restedness 5.3. Of patients who accepted earplugs (59\%), there were improvements to mean sleep quality and quantity (7.6 hours), with $86 \%$ patients rating earplugs helpful. All patients surveyed thought that earplugs should be offered routinely on admission. $70 \%$ of patients were prescribed benzodiazepines or z-drugs as required. Self-reported factors affecting sleep included noise, psychiatric symptoms and medication side effects, with 13 patients mentioning the former. Sound monitoring recorded an average decibel level with a range of $35-75 \mathrm{~dB}$, with peaks reaching $95 \mathrm{~dB}$.

Conclusion. Poor sleep in hospital is widespread. There is a need to understand and address modifiable environmental and ward factors implicated in sleep disturbance within inpatient settings. Pharmacological options for sedation are common, but it is important to focus on alternative options of low-cost, nonpharmacological interventions which promote sleep optimisation and enhance inpatient care.

Developing a local service to improve the provision of palliative care to people who use substances

Vyasa Immadisetty ${ }^{1 \star}$, Natasha Palipane ${ }^{2}$, Tracy Reed ${ }^{1}$, Srirupa Gupta ${ }^{3}$, Beverley Pickett ${ }^{4}$, Fiona McDowall ${ }^{1}$ and Julia Thompson ${ }^{4}$

${ }^{1}$ Essex Partnership University NHS Foundation Trust; ${ }^{2}$ London School of Hygiene and Tropical Medicine, Farleigh Hospice; ${ }^{3}$ Mid and South Essex NHS Foundation Trust and ${ }^{4}$ East Suffolk and North Essex NHS Foundation Trust

${ }^{*}$ Corresponding author.

doi: 10.1192/bjo.2021.526

Aims. To develop a new service model that engages and improves the provision of palliative care to PWUS.

Background. Although people who use substances (PWUS) continue to die prematurely compared to the general population, they are now more likely to die from chronic diseases rather than from drug-related deaths. Challenges to providing palliative care to PWUS include delayed care-seeking behaviours, complex drug interactions and lack of healthcare provider experience.

Method. An informal factorial analysis elucidated population needs through: a review of local databases to estimate the prevalence of palliative need, a thematic review into the deaths of patients in specialist drug services and, a survey of health practitioners' knowledge and attitudes. These informed the service development phase which involves three key components: 1 . A systems approach to increasing patient identification, incorporating key multi-disciplinary stakeholders across hospital- and community-based care 2. Targeted training of healthcare providers and 3. Medicines management for symptom palliation amidst concurrent substance use (including substitution treatments).

Result. The palliative needs of PWUS are under-identified: the local substance service was not partaking in the palliative referral pathway. Only 7\% of a local hospice's annual caseload was recognised as having substance use problems. The care pathway was described as fragmented. Although $>80 \%$ of surveyed palliative care practitioners had experienced caring for PWUS, confidence 